Pacific Journal of Mathematic 


\title{
WHEN IS A POINT BOREL?
}

\author{
P. W. Harley, III AND G. F. MCNulty
}

Let $X$ be a topological space. We investigate the question: When is a point (of $X$ ) Borel? In relation to this, we establish the equivalence of (a) Each point (singleton) is Borel, (b) Each point is the intersection of closed set and a $G_{\delta}$, (c) The derived set of each point is Borel, (d) The derived set of each point is an $F_{\sigma}$, (e) The derived set of each subset is Borel, and (f) The derived set of each subset is an $F_{\sigma}$. Conditions (a), (b), (c), and (d) are also equivalent for a fixed point. As a separation axiom (a) is shown to lie strictly between $T_{1}$ and $T_{0}$. A number of examples are given and the work of other authors discussed.

o. Introduction. Consideration of the question posed in the title for a particular case led to the development of the theorem below.

THEOREM 0.1. The following are equivalent conditions for a topological space $X$.

(a) Each point (singleton) of $X$ is a Borel set.

(b) Each point of $X$ is the intersection of a closed set and a $G_{\delta}$ set.

(c) The derived set of each point of $X$ is a Borel set.

(d) The derived set of each point of $X$ is an $F_{\sigma}$ set.

(e) The derived set of each subset of $X$ is a Borel set.

(f) The derived set of each subset of $X$ is an $F_{\sigma}$ set.

The initial discovery was the implication $(a) \Rightarrow(b)$. Using it, one can show directly ${ }^{1}$ that the $T_{0}$ separation axiom is satisfied if each point is Borel, with the latter condition certain for $T_{1}$ spaces.

In [1], C. E. Aull and W. J. Thron introduce and study a number of separation axioms between $T_{0}$ and $T_{1}$, each of which is classified by some property of derived sets. In Theorem 3.1 of [1], they prove that $\{p\}^{\prime}$ is closed (which is taken as a separation axiom, $T_{D}$ ) if and only if there is a closed set $F$ and an open set $U$ such that $\{p\}=$ $F \cap U$, for all $p \in X$. With this as a catalyst, the equivalence of (b) and (d) is established and "each point is Borel" is fit into the classification scheme of Aull and Thron as follows:

1 We shall do it differently. 
a space $X$ is

$T_{1}$ if and only if $\{p\}^{\prime}$ is empty, for each $p \in X$.

$T_{D}$ if and only if $\{p\}^{\prime}$ is closed, for each $p \in X$.

Each point of $X$ is Borel if and only if $\{p\}^{\prime}$ is an $F_{\sigma}$ set, for each $p \in X$.

$T_{0}$ if and only if $\{p\}^{\prime}$ is a union of closed sets, for each $p \in X .^{2}$

The implication $(b) \Rightarrow(f)$ was suggested to us by the mention in [1] of an observation of C. T. Yang (see [2, p. 56]) to the effect that the derived set of every subset of $X$ is closed if and only if the derived set of every point in $X$ is closed.

The equivalence of (c) and (d) or (e) and (f) might be interpreted as saying that the attempt to classify separation axioms by the Borel complexity of derived sets collapses to just three cases: $T_{1}, T_{D}$, and "each point is Borel".

Section one is devoted to a proof of Theorem 0.1.

In Section two we assemble some other results concerning the property "each point is Borel". First, we show it is necessarily observed in each first countable $T_{0}$ space. Next, an example is given of a $T_{0}$ space in which no point is Borel $^{3}$. Finally, the property (thus each of (a)-(f)) is shown to be countably (but not generally) productive, hereditary, and not preserved by quotient (even closed) maps. In all of these arguments it is the equivalence $(a) \Leftrightarrow(b)$ that is used.

For purposes of application, we remark that properties (a), (b), (c), and (d) at a fixed point $p$ are equivalent.

1. A proof of Theorem 0.1. Fix a space $X$. As is well known the Borel sets in $X$ can be specified by the following recursion.

$\mathscr{B}_{0}$ is the collection of all sets which are either closed or open. $\mathscr{B}_{\alpha+1}$ consists of the unions and intersections of countable collections of members of $\mathscr{B}_{\alpha}$, whenever $\alpha$ is an ordinal with $\alpha<\omega_{1}$. $\mathscr{B}_{\lambda}=\mathbf{U}_{\alpha<\lambda} \mathscr{B}_{\alpha}$ for each limit ordinal $\lambda \leqq \omega_{1}$.

The Borel subsets of $X$ are just the members of $\mathscr{B}_{\omega_{1}}$.

Denote by $\mathscr{C}$ the collection of those subsets of $X$ that can be obtained as the intersection of a closed set and a $G_{i}$ set. It is easy to see that $\mathscr{C}$ is closed under countable intersections and that $\mathscr{B} 0 \subset \mathscr{C} \subset \mathscr{B}_{1}$.

The following is the main lemma.

2 Aull and Thron state this last equivalence as Theorem 2.3 (e) in [1]. They entertain a number of other separation axioms as well.

${ }^{3}$ In connection with this, we remark that of the fourteen examples in [3] of $T_{0}$ spaces that are not $T_{1}$, all of them enjoy the above property and twelve are even $T_{D}$. 
Lemma 1.0. For every ordinal $\alpha<\omega_{1}$ and every $S \in \mathscr{B}_{\alpha}$ if $p \in S$ then there exists $C \in \mathscr{C}$ such that $p \in C \subset S$.

Proof. We proceed by transfinite induction on $\alpha$. When $\alpha=0$ we can let $C=S$. Our induction hypothesis is

For every ordinal $\beta<\alpha$ and every $T \in \mathscr{B}_{\beta}$ if $p \in T$ then there exists $C \in \mathscr{C}$ with $p \in C \subset T$.

Now let $S \in \mathscr{B}_{\alpha}$ with $p \in S$. There are two cases.

Case (i). For each $i \in \omega$, there is an ordinal $\gamma(i)<\alpha$ and sets $B_{i} \in \mathscr{B}_{\gamma^{(i)}}$ such that $S=\bigcup_{i \in \omega} B_{i}$. We may assume $p \in B_{0}$. According to our induction hypothesis there is $C \in \mathscr{C}$ with $p \in C \subset B_{0} \subset$ $\mathrm{U}_{i \in \omega} B_{i}=S$.

Case (ii). For each $i \in \omega$, there is an ordinal $\gamma(i)<\alpha$ and sets $B_{i} \in \mathscr{B}_{\gamma^{(i)}}$ such that $S=\bigcap_{i \in \omega} B_{i}$. By our induction hypothesis for each $i \in \omega$ pick $C_{i} \in \mathscr{C}$ with $p \in C_{i} \subset B_{i}$. Put $C=\bigcap_{i \in \omega} C_{i}$. Then $C \in \mathscr{C}$ and $p \in C \subset \bigcap_{i \in \omega} B_{i}=S$.

In either case the induction is complete and the lemma is established.

Proof of Theorem 0.1. We shall establish the implications

$$
(\mathrm{a}) \Longrightarrow(\mathrm{b}) \Longrightarrow(\mathrm{d}) \Longrightarrow(\mathrm{f}) \Longrightarrow(\mathrm{e}) \Longrightarrow(\mathrm{c}) \Longrightarrow(\mathrm{a}) \text {. }
$$

(a) $\Rightarrow(b)$ : If $\{p\}$ is a Borel set, then for some $\alpha<\omega_{1},\{p\} \in \mathscr{B}_{\alpha}$. By Lemma 1.0 there is $C \in \mathscr{C}$ such that $p \in C \subset\{p\}$ and so $\{p\}=$ $C \in \mathscr{C}$.

$(\mathrm{b}) \Rightarrow(\mathrm{d})$ : Choose a closed set $F$ and a countable collection $\left\{U_{i}: i \in \omega\right\}$ of open sets such that $\{p\}=F \cap\left(\bigcap_{i \in \omega} U_{i}\right)$. We may assume that $F=\{\bar{p}\}$. Moreover, we have $\{p\}^{\prime}=\{\overline{p\}} \sim\{p\}$. Clearly, $\{p\}^{\prime}=\bigcup_{i \in \omega}\left[\left(X \sim U_{i}\right) \cap \overline{\{p\}}\right]$, whereupon $\{p\}^{\prime}$ is an $F_{\sigma}$ set.

(d) $\Rightarrow(f)$ : Let $A$ be a nonempty subset of $X$. We must prove that $A^{\prime}$ is an $F_{\sigma}$ set. For each point $p \in A \sim A^{\prime}$ we choose an open neighborhood $U_{p}$ of $p$ satisfying

(i) $U_{p} \cap A=\{p\}$.

Using (i), it is easy to see that the relation

(ii) $U_{p} \cap A^{\prime} \subset\{p\}^{\prime}$

holds for each $p \in A \sim A^{\prime}$. Next we assert that

(iii) If $p \neq q$, then $U_{p} \cap U_{q} \cap \bar{A}$ is empty, for all $p, q \in A \sim A^{\prime}$. 
To see this, assume $r \in U_{p} \cap \bar{A}$. If $r=p$, then $r \notin U_{q}$ by (i). Otherwise $r \in U_{p} \cap A^{\prime}$, by (i). Accordingly, $r \in\{p\}^{\prime}$ by (ii). But $U_{q}$ is an open set with $p \notin U_{q}$. Consequently $r \notin U_{q}$.

Now let $U=\bigcup\left\{U_{p}: p \in A \sim A^{\prime}\right\}$. For each $p \in A \sim A^{\prime}$, choose a sequence $\left\langle F_{p, i}: i \in \omega\right\rangle$ of closed sets such that $\{p\}^{\prime}=\bigcup_{i \in \omega} F_{p, i}$. Let $B_{i}=\cup\left\{U_{p} \cap F_{p, i}: p \in A \sim A^{\prime}\right\}$. On the basis of the foregoing, it is not difficult to see that both

$$
\text { (iv) } A^{\prime} \cap U=\bigcup_{i \in \omega} B_{i}
$$

and

(v) $\left(A \sim A^{\prime}\right) \cap\left(\bigcup_{i \in \omega} B_{i}\right)$ is empty.

We now demonstrate the validity of (vi) below.

(vi) $B_{i}=\bar{B}_{i} \cap U$.

Since $B_{i} \subset U$ by definition, it is clear that $B_{i} \subset \bar{B}_{i} \cap U$. Suppose that $x \in \bar{B}_{i} \cap U$. Choose $p \in A \sim A^{\prime}$ such that $x \in U_{p}$. If $q \in A \sim A^{\prime}$ with $q \neq p$, then $U_{p} \cap U_{q} \cap F_{q, i} \subset U_{p} \cap U_{q} \cap \bar{A}$. But $U_{p} \cap U_{q} \cap \bar{A}$ is empty according to (iii). Since $x \in \bar{B}_{i}$ and $U_{p}$ is a neighborhood of $x$, it must be that $x \in \overline{U_{p} \cap F_{p, i}}$, whereupon $x \in F_{p, i}$. So $x \in U_{p} \cap F_{p, i} \subset B_{i}$ and (vi) is established.

Since $A \sim A^{\prime} \subset U$, in view of (vi) we can extend (v) to

(vii) $\left(A \sim A^{\prime}\right) \cap\left(\bigcup_{i \in w} \bar{B}_{i}\right)$ is empty.

Since $A^{\prime} \sim U=\bar{A} \sim U$, we have the equations

$$
\begin{aligned}
A^{\prime} & =\left(A^{\prime} \cap U\right) \cup\left(A^{\prime} \sim U\right) \\
& =\left(A^{\prime} \cap U\right) \cup(\bar{A} \sim U)
\end{aligned}
$$

and then

$$
\text { (viii) } A^{\prime}=\left(\bigcup_{i \in \omega} B_{i}\right) \cup(\bar{A} \sim U) .
$$

But using the relation $\bar{B}_{i} \subset \bar{A}$, (vi), and (viii) we have

$$
\text { (ix) } A^{\prime}=\left(\bigcup_{i \in w} \bar{B}_{i}\right) \cup(\bar{A} \sim U)
$$

and therefore $A^{\prime}$ is an $F_{\sigma}$ set.

$(\mathrm{f}) \Rightarrow(\mathrm{e})$ and $(\mathrm{e}) \Longrightarrow(\mathrm{c})$ are obvious.

(c) $\Rightarrow$ (a): This follows from the equation $\{p\}=\{\bar{p}\} \sim\{p\}^{\prime}$. This completes the proof of theorem 0.1 . 
2. Other results. First we make the observation below.

THEOREM 2.0. Every point of a first countable $T_{0}$ space is Borel.

Proof. Let $X$ be a first countable $T_{0}$ space and $p \in X$ with an (open) neighborhood base $\left\langle U_{i}: i \in \omega\right\rangle$. Put $G=\bigcap_{i \in \omega} U_{i}$. For each $x \in G \sim\{p\}$ pick an open neighborhood $V_{x}$ of $x$ with $p \notin V_{x}$. Let $F=X \sim \bigcup\left\{V_{x}: x \in G \sim\{p\}\right\}$. Notice that $\{p\}=F \cap G$ and accordingly $\{p\}$ is a Borel set.

EXAMPLe 2.1.4 A $T_{0}$ space in which no point is Borel.

We construct a set $X$ and a strict dense linear ordering $R$ on $X$ without endpoints such that

(*) If $Y$ is a countable subset of $X$ and $p \in X$ such that $y R p$ for all $y \in Y$, then there is $q \in X$ with $q R p$ and $y R q$ for $y \in Y$.

Once such a set $X$ and ordering $R$ have been constructed we define a topology by calling $U \subset X$ a basic open set if and only if there is $p \in X$ with $U=\{q: p R q$ and $q \in X\}$.

$X$ and $R$ are obtained as direct limits on the basis of the following recursion over the ordinals $\alpha$.

$X_{0}=\{0\}$

$X_{\alpha+1}=X_{\alpha} \times\{-1,0,1\}$
$R_{0}$ is empty

$R_{\alpha+1}$ is the lexicographic ordering of $X_{\alpha}$ induced by $R_{\alpha}$ and the usual ordering $-1<0<1$ on $\{-1,0,1\}$.

Note. $X_{\alpha}$ can be identified with $X_{\alpha} \times\{0\}$, in which case $R_{\alpha}$ is identified with $R_{\alpha+1}$ restricted to $X_{\alpha} \times\{0\}$.

If $\lambda$ is a limit ordinal, then let

$$
X_{\lambda}=\bigcup_{\alpha<\lambda} X_{\alpha} \quad R_{\lambda}=\bigcup_{\alpha<\lambda} R_{\alpha} .
$$

We let $X=X_{\omega_{1}}$ and $R=R_{\omega_{1}}$.

Evidently $R$ is a dense linear ordering of $X$ without endpoints. $\langle X, R\rangle$ fulfills $\left(^{*}\right)$ since $Y \cup\{p\} \in X_{\alpha}$ for some $\alpha<\omega_{1}$ and by taking $q=(p,-1) \in X_{\alpha+1}$ we obtain

$$
(y, 0) R_{\alpha+1} q R_{\alpha+1}(p, 0) \text { for all } y \in Y .
$$

${ }^{4}$ A simpler example of a $T_{0}$ space in which some point is not Borel is $\omega_{1}+1$ endowed with the right ray topology. The space constructed here has cardinality $\aleph_{1}$. Example 2.3 below also provides a $T_{0}$ space in which no point is Borel, but it is of cardinality $2 \aleph_{1}$. 
Since $(y, 0)$ is identified with $y$ and $(p, 0)$ is identified with $p$ (in $X$ ) $(*)$ is verified. ${ }^{5}$

Now let $p \in X$. In the (right ray) topology described above $\overline{\{p}\}=\{q: q R p$ or $q=p\}$. Let $\left\langle U_{i}: i \in \omega\right\rangle$ be any countable system of basic open neighborhoods of $p$. Accordingly for each $i \in \omega$ pick $r_{i} \in X$ with $U_{i}=\left\{t: t \in X\right.$ and $\left.r_{i} R t\right\}$. In particular $r_{i} R p$ for all $i \in \omega$. Consequently, by $\left({ }^{*}\right)$ there exists $x \in X$ such that $x R p$ and $r_{i} R x$ for all $i \in \omega$. Therefore $x \in \overline{\{p\}} \cap \bigcap_{i \in \omega} U_{i}$. Since $x \neq p$, we conclude that $\{p\} \neq \overline{\{p\}} \cap \bigcap_{i \in \omega} U_{i}$ and so that $\{p\}$ is not the intersection of any closed set and any $G_{o}$ set. Thus, by Theorem $0.1((a) \Leftrightarrow(b))$ no point of $X$ is Borel. $X$ is clearly $T_{0}$.

THEOREM 2.2. The property "each point is Borel" is hereditary and countably productive.

Proof. The first statement is an easy consequence of Theorem $0.1((a) \Leftrightarrow(b))$ which we leave to the reader.

As for the second, again we use Theorem $0.1((a) \Leftrightarrow(b))$. Let $\left\langle X_{i}: i \in \omega\right\rangle$ be a sequence of spaces in which each point is Borel and let $p=\left\langle p_{i}: i \in \omega\right\rangle$ be a point in the product space $X=\prod_{i \in \omega} X_{i}$. For each $i \in \omega$ represent $\left\{p_{i}\right\}=F_{i} \cap\left(\bigcap_{j \in \omega} U_{i, j}\right)$ where $F_{i}$ is closed in $X_{i}$ and $U_{i, j}$ is open in $X_{i}$, for each $i, j \in \omega$. It follows that

$$
\{p\}=\bigcap_{i \in \omega}\left[\pi_{i}^{-1}\left(F_{i}\right) \cap\left(\bigcap_{j \in \omega} \pi_{i}^{-1}\left(U_{i, j}\right)\right)\right],
$$

whereupon every point in $X$ is Borel. The case of finite products is similar.

EXAMPLE 2.3. A space $X$ in which each point is Borel and nevertheless every finite indiscrete space is a closed image of $X$ and $X^{k}$ has no Borel points provided $\kappa>\aleph_{0}$.

We take $X$ to be the set of real numbers ${ }^{6}$ endowed with the right ray topology. As $X$ is a first countable $T_{0}$ space, we know that each point of $X$ is Borel.

Let $Y=\left\{y_{0}, y_{1}, \cdots, y_{n-1}\right\}$ be a finite indiscrete space. Partition

${ }^{5}$ All of the ambiguities of this construction can be avoided at the expense of involving the relatively complicated construction of the limit of a system of relations directed by a system of embeddings. Heuristically, the argument is: "start with a point and a sharp knife. Chop each point into left, middle, and right pieces. Do this $\boldsymbol{\aleph}_{1}$ times."

${ }^{6}$ We could use the rationals as easily; even the Sierpinski space $S=\{0,1\}$, whose open sets are $\{0,1\},\{1\}$, and the empty set, has the property that $S \boldsymbol{N}_{1}$ has no Borel points. 
the negative integers into $n$ infinite sets $X_{0}, X_{1}, \cdots, X_{n-1}$ and define $f: X \rightarrow Y$ by

$$
f(x)= \begin{cases}y_{i} & \text { if } x \in X_{i} \\ y_{0} & \text { otherwise }\end{cases}
$$

Then every closed set of $X$ (a closed left ray) maps onto $Y$. Thus $f$ is closed. Since $Y$ is indiscrete $f$ is continuous.

As for the second assertion about $X$, let $\kappa>\aleph_{0}$ and $p \in X^{\kappa}$. Note that $p$ is a function with domain $\kappa$ and range included in $X$. It is easy to see that $\{\bar{p}\}=\left\{q: q \in X^{\kappa}\right.$ and $q_{\alpha} \leqq p_{\alpha}$ for all $\left.\alpha \in \kappa\right\}$. If $\left\langle U_{i}: i \in \omega\right\rangle$ is a countable sequence of basic open sets in $X^{k}$, it is easy to see that $\left\{\overline{\{p} \cap \bigcap_{i \in \omega} U_{i} \neq\{p\}\right.$. Thus, any pepresentation of $\{p\}$ as the intersection of a closed set and a $G_{\delta}$ set is impossible. An application of Theorem 0.1 now finishes the argument.

\section{REFERENCES}

1. C. E. Aull and W. J. Thron, Separation axioms between $T_{0}$ and $T_{1}$, Indag. Math., 24 (1962), 26-37.

2. J. L. Kelley, General Topology, Van Nostrand, New York, 1955.

3. L. A. Steen and J. A. Seebach, Counterexamples in Topology, Holt, Rinehart and Winston, Inc., New York, 1970.

Received July 29, 1977.

University of South Carolina

Columbia, SC 29208 



\section{PACIFIC JOURNAL OF MATHEMATICS}

\section{EDITORS}

RICHARD ARENS (Managing Editor)

University of California

Los Angeles, California 90024

C. W. CURTIS

University of Oregon

Eugene, OR 97403

C. C. MOORE

University of California

Berkeley, CA 94720
J. DugundjI

Department of Mathematics University of Southern Californı Los Angeles, California 90007

R. Finn and J. Milgram Stanford University Stanford, California 94305

ASSOCIATE EDITORS

E. F. BECKENBACH

B. H. NeUmanN

F. WOLF

K. YoSHIDA

\section{SUPPORTING INSTITUTIONS}

UNIVERSITY OF BRITISH COLUMBIA CALIFORNIA INSTITUTE OF TECHNOLOGY

UNIVERSITY OF CALIFORNIA

MONTANA STATE UNIVERSITY

UNIVERSITY OF NEVADA, RENO

NEW MEXICO STATE UNIVERSITY

OREGON STATE UNIVERSITY

UNIVERSITY OF OREGON
UNIVERSITY OF SOUTHERN CALIFORNIA

STANFORD UNIVERSITY

UNIVERSITY OF HAWAII

UNIVERSITY OF TOKYO

UNIVERSITY OF UTAH

WASHINGTON STATE UNIVERSITY

UNIVERSITY OF WASHINGTON 


\section{Pacific Journal of Mathematics}

\section{Vol. 80, No. $1 \quad$ September, 1979}

Jeroen Bruijning and Jun-iti Nagata, A characterization of covering dimension by

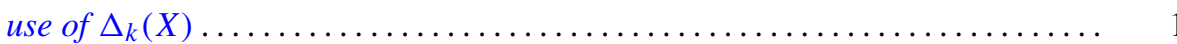

John J. Buoni and Albert Jonathan Klein, On the generalized Calkin algebra ...... 9

Thomas Ashland Chapman, Homotopy conditions which detect simple homotopy

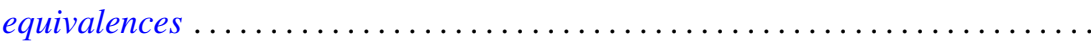

John Albert Chatfield, Solution for an integral equation with continuous interval

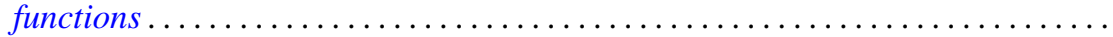

Ajit Kaur Chilana and Ajay Kumar, Spectral synthesis in Segal algebras on

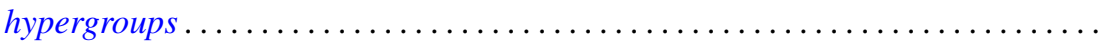

Lung O. Chung, Jiang Luh and Anthony N. Richoux, Derivations and

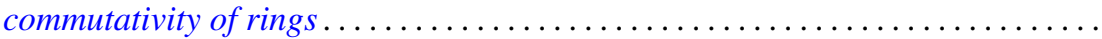

Michael George Cowling and Paul Rodway, Restrictions of certain function spaces to closed subgroups of locally compact groups .....................

David Dixon, The fundamental divisor of normal double points of surfaces........

Hans Georg Feichtinger, Colin C. Graham and Eric Howard Lakien,

Nonfactorization in commutative, weakly selfadjoint Banach algebras . . . . . . .

Michael Freedman, Cancelling 1-handles and some topological imbeddings ....... .

Frank E., III Gerth, The Iwasawa invariant $\mu$ for quadratic fields . . . . . . . . . . . . . .

Maurice Gilmore, Three-dimensional open books constructed from the identity

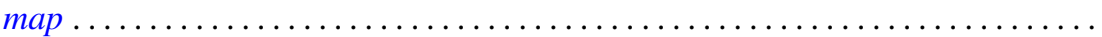

Stanley P. Gudder, A Radon-Nikodým theorem for $*$-algebras .

Peter Wamer Harley, III and George Frank McNulty, When is a point Borel? .

Charles Henry Heiberg, Fourier series with bounded convolution powers . .

Rebecca A. Herb, Characters of averaged discrete series on semisimple real Lie

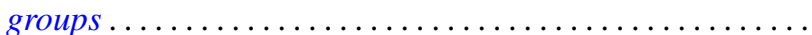

Hideo Imai, On singular indices of rotation free densities . .

Sushil Jajodia, On 2-dimensional CW-complexes with a single 2-cell . . .

Herbert Meyer Kamowitz, Compact operators of the form $u C_{\varphi}$

Matthew Liu and Billy E. Rhoades, Some properties of the Chebyshev method...

213

George Edgar Parker, Semigroups of continuous transformations and generating

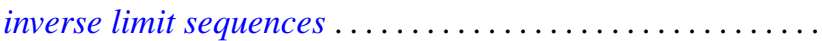

Samuel Murray Rankin, III, Oscillation results for a nonhomogeneous

equation ...

Martin Scharlemann, Transverse Whitehead triangulations ...

Gary Joseph Sherman, A lower bound for the number of conjugacy classes in a

finite nilpotent group

Richard Arthur Shoop, The Lebesgue constants for $\left(f, d_{n}\right)$-summability .

Stuart Jay Sidney, Functions which operate on the real part of a uniform

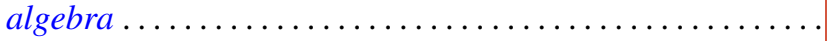

Tim Eden Traynor, The group-valued Lebesgue decomposition 